\title{
Workplace diaries promoting reflective practice in radiation therapy.
}

\section{Introduction}

In order to stay current with the changing techniques and emerging technology and the expansion of roles it is important that radiation therapist's development a commitment to life long learning (LLL) and continuing professional development (CPD) in their everyday practice [1-5]. Common methods for undertaking LLL and CPD can be via structured programs, whilst a complementary approach is the incorporation of self guided reflective practice and thinking. Practising reflectively can assist the practitioner to monitor aspects of their practice, encourage professional development, personal growth and to evaluate the currency of their skills. One method that can be used to document reflective thinking is by using a written reflective journal.

Although a small amount of literature describes the informal reflective nature of daily radiation therapy practice, not all practitioners are able to reflect and certainly not to the same level [6]. Practitioners can improve their skills in reflective thinking, writing and ultimately reflective practice through education and awareness of the concepts central to reflective practice and frameworks available to guide reflective journaling.

The purpose of this paper is to review the literature on reflective practice and frameworks for reflective journaling in allied health and nursing, and to provide a guide for reflective journaling or workplace diaries for radiation therapists. 
This will be done by discussing the role of reflective practice, as well as define concepts central to reflective journaling or workplace diaries. Finally the paper will offer practical advice to increase staff knowledge and skills in the use of reflective workplace diaries.

\section{Defining reflective practice}

To encourage and foster reflective practice as a self learning tool in the professional practice setting there needs to be a definition that radiation therapists can use as a starting point. There are three key elements to the concept of reflection; reflection surrounds the process of learning, reflection is purposeful in its intent, and reflection addresses topics or problems where there is no obvious solution and reflective processing is required [7]. The literature presents an array of definitions for the process of reflection as demonstrated in table 1 . The authors of this current paper have defined reflective practice as:

"Involving self, a process that is undertaken in response to a positive or negative event that may be initiate consciously or subconsciously, that requires analysis to provide an answer or insight."

\section{INSERT TABLE 1}

\section{Radiation therapy clinical practice and reflection}

There is little literature that discusses the concepts and processes of reflection within radiation therapy, compared to the amount of literature detailing the use of reflection within the nursing profession. Like most 
professionals those working in Medical Radiation Science (MRS) have the potential to become habitual in their practice. Baird [8] accurately describes this repetitive state of practice to be a 'non-learning' situation. To avoid becoming entrapped in this mode of practice, Baird highlights that it is essential for practitioners to be aware and in some cases critical of their thought process [8].

Newnham [9] discusses the advantages of reflection in the radiation therapy field and the ways in which reflection is already integrated into daily practice. He highlights the importance of radiation therapists knowing and feeling confident in their specialised knowledge especially on a multidisciplinary stage, suggesting that a lack of reflection may have inhibited this discovery in the past.

Benefits of incorporating reflection into radiation therapist's professional practice can include:

- Introduction of a structured tool or process for therapists to express radiation therapy knowledge of everyday practice

- Enabling therapists to share knowledge with others [9] to benefit practice, the profession and patient outcomes

- Enabling individuals or groups to reflect, verify and examine clinical actions, optimise work practice, improve inter-professional relationship

- Incorporating formal or structured reflection into the clinical environment that allow techniques and developments to be constantly evaluated and adapted, promoting best practice [4]. In daily decisions 
radiation therapists are constantly and repeatedly evaluating clinical parameters as indicators of accuracy in highly complex situations

- Providing a method to review or improve decision making abilities

- Exploring areas of special interest where advanced practice may occur and

- Realising career desires and aspirations allowing for career mapping or planning.

All radiation therapists decisions in the areas of planning, treatment and simulation are subject to peer reviewed quality assurance assessment, which may indicate that this repetitive, yet informal and mostly undocumented reflective practice environment may be unique in the health profession [9]. In addition to these highly clinical and empirically based decisions there are many elements to daily radiation therapy practice that are centred on the implicit knowledge that only comes from heuristic experience (trial and error). It is in these decisions that can not always be answered by empirical evidence, nor rationalisation and where there is no obvious answer, that tacit knowledge (knowledge held in the mind that can not always be easily accessed) and the use of reflection to access it, is vitally important in the practice of radiation therapy. These decisions alone may not have large clinical impact, they are more often than not decisions concerning patient stability, localisation problems, difficult treatment plans or a puzzling patient presentation, but all have an impact on workflow and ultimately patient experience. It is the role of reflection in these situations that White [4], 
suggests could support existing practice and if necessary provide a process to facilitate change.

\section{CPD and reflection}

CPD is essential to the currency of clinical skills. It can take on many forms, one of which is structured formal education such as post graduate academic programs, conferences and national/international courses. Structured CPD can also include in-service training and workshops offered within departments. More informal CPD can include debriefing sessions, brain storming exercises and learning though daily work. Often informal CPD is on an individual basis and can include journal reading and workplace diaries or journaling.

Completing a professional journal or workplace diary is a very personal and individual form of reflective practice. These journals can be based around one of the many reflective models available in the literature or very informally written in the preferred style and format of the individual. There has been much focus on how structured reflection can be incorporated into CPD as a means to enhance and maintain reflective practice in the clinical environment. The Australian Institute of Radiography have incorporated reflective journaling as an element of the professional portfolio program [10].

Similar to other allied health care professions, there is a lack of any large scale research into the ability of reflective frameworks to increase a professional's ability to be reflective or become a reflective practitioner. 
However, reflective practice affords the ability to examine our beliefs and attitudes in dealing with cancer patients and working in the cancer setting, suggesting that reflective practice is a highly intuitive and personal process. This subjectivity makes reflective journaling difficult to investigate with historic quantitative methodologies [4], and can not realistically lend itself to a rationalistic inquiry such a large scale empirical study or trial $[11,12]$. Heath [13] describes the benefits of introducing structured reflective models as unlikely to be quantifiable, however anecdotally he believes practitioners are able to make substantial professional progress with limited reflective practice exercises.

\section{Reflective journaling in radiation therapy}

Journaling or workplace diaries is not the only method available to facilitate reflection, however, in radiotherapy departments where staff and resources are often stretched, journaling to facilitate reflection may minimise the burden on resources [14]. The literature discusses three approaches to reflective writing in clinical practice: reflective journals, significant event analysis and portfolios. There is significant overlap between all these techniques [15], hence this paper only discusses reflective journaling.

Reflective journals can be completed by health professionals at any interval, daily, monthly or randomly. They are an ideal way for them to be actively involved in their learning [14]. Typical academic requirements such as spelling, grammar and syntax assessment are seen as less important, hence enabling those that may be burdened with the restraints of academic prose the freedom to tell their story without criticism [6]. Writing is the closest form 
of communication to natural speech. Often people are able to write and allow their thought to flow in an uninhibited manner, it is this articulation of thought that is said to promote learning and skill development [16-18].

A vast amount of literature has been written over the past three decades that discusses the use of journaling or reflective assignments as a tool to promote reflective thinking skills in the fields of nursing, medicine and allied health sciences [6, 19]. Reflective journals are seen to allow health care workers to record events, document their reactions to them and how this may effect their future practise [20]; as well as explore changes in their attitudes and often identify what it means to be a professional [21], facilitating them to become autonomous self directed learners [22]. There is no consensus that structured reflective models will provide an increase in reflective practice in clinical situations compared to less formal methods of reflection [23, 24], although some authors insist that for reflective writing to assist in learning it is essential to "set parameters or rules for writing" [25, p.43].

\section{Starting a reflective workplace diaries}

Many clinical health professionals are often reluctant to begin writing reflective journals because many do not see the value in writing things down as they are predominantly "doers" and their primary mode of communication is verbal [25] and are unsure how to initiate the process. In everyday practice health practitioners talk to each other, talk to their patients and their families. It is important to remember when promoting reflective journaling the assumption is made that the practitioner is able to reflect, willing to reflect and also willing to make changes as a result of this reflection. If the concepts surrounding 
reflection and reflective journaling are simplified and its relevance to clinical practice clearly described, more radiation therapists may be interested in undertaking it as a CPD activity.

Reflective workplace diaries can be completed at any interval; daily, monthly or after a significant event that the practitioner wants to write about. The length of the entry may vary from a few lines to pages, depending on the topic and impact it had on the author. This diary does not have to be completed in a specific format, it could be a small notebook, loose leaf paper or in electronic form. Stimulus for completing a diary entry can originate from many events, some examples include:

- A patient interaction

- A workshop or conference attendance

- A change in work location due to roster change

- An interesting conversation with a colleague, and

- A critical incident.

By asking simple questions such as, what happened and why? how do I feel about it? what have I learnt from it? The practitioner will be able to begin exploring the topic of the entry in a deep and personal manner.

\section{Reviewing your workplace diaries}

It is unrealistic to expect a large cross section of therapists to be skilled in writing reflectively and then reviewing their work. It is important however for practitioners to review their writing as a means of gaining feedback on their development as a reflective writer and for improvements to clinical practice. 
One simple model that can assist the review of reflective writing is the work of Boud, et al, [26]. This model defines and describes the stages of reflection that could be easily adapted to a personalised framework to support the review reflective journaling in professional practice. Adapted from the work of Boud et.al.[26] table 2 provides a guide and examples to consult when reviewing your workplace diary. This can either be used as a means to audit the diary if it is included in a professional portfolio that may be externally reviewed or simply as a means to gain an insight into the quality of your reflective writing on a personal level.

Reviewing the diary will assist in the progression of reflective writing from low to higher, more critical levels of reflection.

\section{INSERT TABLE 2}

\section{Conclusion}

There are obvious barriers to formalised or documented reflective writing within a RT department, such as time restraints, some staff not knowing how to reflect in such a manner and being apprehensive about documenting personal experiences or emotions [25]. This paper provides radiation therapists with ideas to guide reflective journaling in an attempt to minimise the barriers to workplace diaries and journals within the RT workplace.

With reflective practice becoming an essential skill to incorporate into clinical practice and $\mathrm{CPD}$ in $\mathrm{RT}$, it is important that radiation therapists understand the role and potential of reflection in these areas. Radiation therapists should 
and can adopt reflective diaries into their professional growth without the need for a formal model to guide them. The use of inventories can simplify the process by directing thoughts and words, enabling progression through the reflective process. 


\section{References}

1. Fell, K., Radiography into the millennium. The practice and process of therapeutic radiography: a professional perspective. Synergy, 1999. September: p. 4-6.

2. Radiation therapy education survey results: establishing a professional database. Radiation Therapist: The Journal of the Radiation Oncology Sciences, 1998. 7(1): p. 85-89.

3. Svensson, H. and T.R. Moller, Developments in radiotherapy. Acta Oncol, 2003. 42(5-6): p. 430-42.

4. White, N., Reflective practice in radiotherapy radiography. Synergy, 2003. June: p. 7-9.

5. Hughes, P., Evaluating the impact of continual professional education. Nurse Educ Today, 1990. 10(6): p. 428-36.

6. Paterson, B., Developing and maintaining reflection in clinical journals. Nurse Educ Today, 1995. 15: p. 211-220.

7. Moon, J.A., Reflection in learning \& professional development : theory \& practice. 1999, London: Kogan Page. x, 229.

8. Baird, M., The idea of a reflective practicum: overcoming the dichotomy between academia and the practice setting. Radiography, 1996. 2: p. 119-138.

9. Newnham, J., To reflect or not? Reflective practice in radiation therapy. Journal of Radiotherapy in Practice, 1999. 1(3): p. 109-116.

10. Guide to AIR Continuing Professional Development. (2007). from http://www.air.asn.au/files/uploads/0316_CPD_Guide1.pdf 
11. Hargreaves, J., Using patients: exploring the ethical dimension of reflective practice in nursing education. Journal of Advanced Nursing, 1997. 25(2): p. 223-228.

12. Palmer, A., S. Burns, and C. Bulman, Reflective Practice in Nursing. 1994, Oxford: Blackwell Scientific Publications.

13. Heath, H., Paradigm dialogues and dogma: finding a place for research, nursing models and reflective practice. Journal of Advanced Nursing, 1998. 27(5): p. 288-294.

14. Milinkovic, D. and N. Field, Demystifying the reflective clinical journal. Radiography, 2005. 11(3): p. 175-183.

15. Henderson, E., et al., Conflict and coping strategies: a qualitative study of student attitudes to significant event analysis. Med Educ, 2003. 37(5): p. 438-46.

16. Kerka, S., Journal writing and adult learning: ERIC Digest no. 174, in Journal of writing and adult education. 1996.

17. Murphy, J., Using Focused Reflection and Articulation to Promote Clinical Reasoning: An Evidence-Based Teaching Strategy. Nursing Education Perspectives, 2004. 25(5): p. 226-231.

18. Usher, K., J. Tollefson, and D. Francis, Moving from technical to critical reflection in journaling: an investigation of students' ability to incorporate three levels of reflective writing. Aust J Adv Nurs, 2001. 19(1): p. 15-9.

19. Williams, R., et al., Journal writing to promote reflection by physical therapy students during clinical placements. Physiotherapy Theory and Practise, 2002. 18. 
20. Williams, R. and J. Wessel, Reflective journal writing to obtain student feedback about their learning during the study of chronic musculoskeletal conditions. J Allied Health, 2004. 33(1): p. 17-23.

21. Winnie, L. and C. Palmer, Investigating Effectiveness of Reflective Journaling For Radiation Therapy Students: A student perspective. Canadian journal of medical radiation technology, 2004. 35(1): p. 3-9.

22. Harris, S., G. Dolan, and G. Fairbairn, Reflecting on the use of student portfolios. Nurse Educ Today, 2001. 21(4): p. 278-86.

23. Mackintosh, C., Reflection: a flawed strategy for the nursing profession. Nurse Educ Today, 1998. 18(7): p. 553-7.

24. Burnard, P., Nurse educators' perceptions of reflection and reflective practice: a report of a descriptive study. Journal of Advanced Nursing, 1995. 21: p. 1167-1174.

25. Rolfe, G., D. Freshwater, and M. Jasper, Reflective Writing, in Critical reflection for nursing and the helping professions: a user's guide. 2001, Palgrave: Basingstoke. p. 41-74.

26. Boud, D.J., D. Walker, and R. Keogh, Reflection, turning experience into learning. 1985, London: Kogan Page ;Nichols Pub. 170.

27. Jarvis, P., Reflective Practice and Nursing. Nurse Educ Today, 1992. 12: p. $174-181$. 